\title{
Intermediate Bone Neoplasm
}

National Cancer Institute

\section{Source}

National Cancer Institute. Intermediate Bone Neoplasm. NCI Thesaurus. Code C121926.

A locally aggressive or rarely metastasizing neoplasm that arises from the bone. 